\title{
Efficient intrinsic spin-to-charge current conversion in an all-epitaxial single-crystal perovskite-oxide heterostructure of $\mathrm{La}_{0.67} \mathrm{Sr}_{0.33} \mathrm{MnO}_{3} / \mathrm{LaAlO}_{3} / \mathrm{SrTiO}_{3}$
}

\author{
Shinobu Ohya $\odot,{ }^{1,2,3, *}$ Daisei Araki, ${ }^{1}$ Le Duc Anh $\odot,{ }^{1,2}$ Shingo Kaneta, ${ }^{1}$ Munetoshi Seki, ${ }^{1,3}$ \\ Hitoshi Tabata, ${ }^{1,3}$ and Masaaki Tanaka ${ }^{1,3}$ \\ ${ }^{1}$ Department of Electrical Engineering and Information Systems, The University of Tokyo, \\ 7-3-1 Hongo, Bunkyo-ku, Tokyo 113-8656, Japan \\ ${ }^{2}$ Institute of Engineering Innovation, Graduate School of Engineering, The University of Tokyo, \\ 7-3-1 Hongo, Bunkyo-ku, Tokyo 113-8656, Japan \\ ${ }^{3}$ Center for Spintronics Research Network (CSRN), Graduate School of Engineering, The University of Tokyo, \\ 7-3-1 Hongo, Bunkyo-ku, Tokyo 113-8656, Japan
}

(Received 11 June 2019; revised manuscript received 22 August 2019; published 14 January 2020)

\begin{abstract}
We demonstrate efficient intrinsic spin-to-charge current conversion in a two-dimensional electron gas using an all-epitaxial single-crystal heterostructure of $\mathrm{LaSrMnO}_{3} / \mathrm{LaAlO}_{3} / \mathrm{SrTiO}_{3}$, which can suppress spin scattering and give us an ideal environment to investigate intrinsic spin-charge conversion. With decreasing temperature to $20 \mathrm{~K}$, the spin-to-charge conversion efficiency is drastically enhanced to $+6.7 \mathrm{~nm}$. Our band-structure calculation well reproduces this behavior and predicts further enhancement by controlling the density and relaxation time of the carriers.
\end{abstract}

DOI: 10.1103/PhysRevResearch.2.012014

Recent observations of conversion phenomena between spin and charge currents promise a substantial reduction of power consumption in next-generation high-speed spintronics devices such as spin-orbit-torque magnetoresistive randomaccess memories [1]. While this conversion is well known to occur in heavy metals [2-4], recent studies have shown that it occurs also at various interfaces such as $\mathrm{Ag} / \mathrm{Bi}, \mathrm{Fe} / \mathrm{Ge}$, and $\mathrm{Ag} / \alpha-\mathrm{Sn}$ [5-9]. At these interfaces, the Rashba spin-orbit interaction and resulting spin splitting of the Fermi surface appear due to the broken space-inversion symmetry, causing a spin-to-charge current conversion that is known as the inverse Edelstein effect (IEE) [10-12].

Very recently, a giant IEE was observed [13] in a twodimensional electron gas (2DEG) formed at the interface between insulating perovskite oxides $\mathrm{LaAlO}_{3}$ (LAO) and $\mathrm{SrTiO}_{3}$ (STO) $[14,15]$. The strong Rashba spin-orbit interaction at LAO/STO, which can be modulated by a gate voltage, makes this system very attractive for controllable efficient spin-charge conversion [16-18]; however, previously reported results for spin-charge conversion at LAO/STO are divergent, which cannot be explained by the difference of the transport properties of the $2 \mathrm{DEG}$, and a unified understanding of the intrinsic mechanism of the spin-charge conversion is still lacking [13,19-21]. For example, in Ref. [13], a large conversion efficiency, the so-called inverse Edelstein length $\lambda_{\text {IEE}}$, of up to $-6.4 \mathrm{~nm}$ was observed at $7 \mathrm{~K}$. This value is

\footnotetext{
*ohya@cryst.t.u-tokyo.ac.jp

Published by the American Physical Society under the terms of the Creative Commons Attribution 4.0 International license. Further distribution of this work must maintain attribution to the author(s) and the published article's title, journal citation, and DOI.
}

much larger than that reported for $\mathrm{Ag} / \mathrm{Bi}\left(\lambda_{\mathrm{IEE}}=0.3 \mathrm{~nm}\right)$. Meanwhile, in Refs. [19,20], the conversion signal strongly decreases to zero with decreasing temperature. The reason for the completely different behavior is not clear at present, but this is likely attributed to inelastic transport of the spin current, which is predicted to reduce the conversion signal, especially at low temperature. This inelastic spin transport is thought to be related to the crystal quality of samples. In metal systems, the interface quality is known to have a large influence on the conversion efficiency [22,23]. In previous studies on spin-charge conversion at LAO/STO [13,19-21], however, amorphous or polycrystalline ferromagnetic films deposited by sputtering were used for the ferromagnetic layer, which may cause strong spin scattering, especially at the interface between the ferromagnetic layer and LAO. In this Rapid Communication, to exploit the intrinsic IEE in the LAO/STO system, we focus on an all-epitaxial single-crystal heterostructure of $\mathrm{La}_{0.67} \mathrm{Sr}_{0.33} \mathrm{MnO}_{3}$ (LSMO)/LAO/STO. LSMO is a strongly correlated half-metallic ferromagnetic-perovskite oxide that can be epitaxially grown on STO due to the small lattice mismatch of $\sim 0.8 \%$. LSMO is thus an ideal candidate to explore the efficient spin injection and intrinsic spin-charge conversion at the LAO/STO interface.

For the experiments, we have prepared a sample composed of $\mathrm{La}_{0.67} \mathrm{Sr}_{0.33} \mathrm{MnO}_{3}$ [30 unit cells (u.c.) $=$ $12 \mathrm{~nm})] / \mathrm{La}_{(1-\delta)} \mathrm{Al}_{(1+\delta)} \mathrm{O}_{3}(\mathrm{LAO}, 2$ u.c. $=0.8 \mathrm{~nm})$ grown on a $\mathrm{TiO}_{2}$-terminated $\mathrm{SrTiO}_{3}(001)$ substrate via molecular beam epitaxy (MBE) [Fig. 1(a)]. We used a shuttered growth technique with fluxes of $\mathrm{La}, \mathrm{Sr}, \mathrm{Mn}$, and $\mathrm{Al}$ supplied by Knudsen cells. The LAO and LSMO layers were grown at $730{ }^{\circ} \mathrm{C}$ with a background pressure of $2 \times 10^{-4} \mathrm{~Pa}$ due to a mixture of oxygen $(80 \%)$ and ozone $(20 \%)$. As shown later, the thickness of 2 u.c. of LAO is large enough to form a $2 \mathrm{DEG}$ at the LAO/STO interface because of the presence of the 
(a)

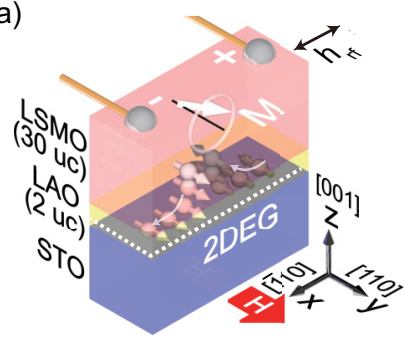

(c)

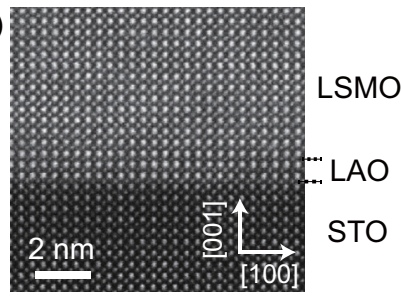

(b)
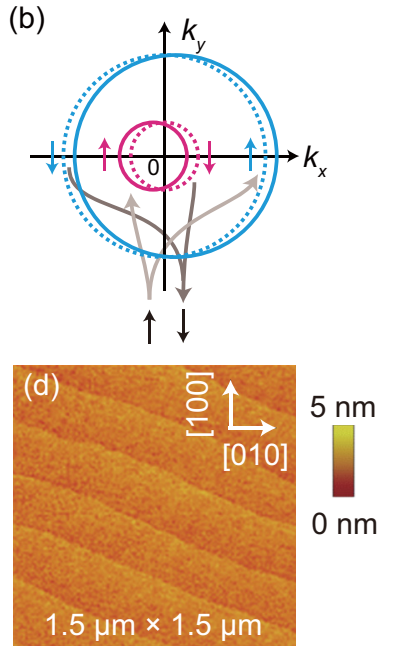

FIG. 1. (a) Schematic illustration of the (001)-oriented fullepitaxial multilayer structure of LSMO/LAO grown on an STO (001) substrate. The sample size is $2 \times 1 \mathrm{~mm}$. In the electron-spinresonance system, a radio-frequency magnetic field $h_{\mathrm{rf}}$ was applied along the [110] $(x)$ direction of the sample. The static magnetic field $\mu_{0} H$ was applied along the [110] (y) axis. Here, $M$ represents the magnetization of LSMO. (b) Principle of spin-to-charge conversion via the inverse Edelstein effect; the spin current injected into the LAO/STO interface moves the outer and inner Fermi circles, generating a charge current in the $x$ direction. Here, the dotted and solid lines are the original Fermi circles and the ones after a spin current is injected, respectively. (c) Scanning-transmissionelectron microscope-lattice image of the LSMO (30 u.c.)/ LAO (2 u.c.)/STO heterostructure (sample A) projected along the [010] axis. (d) Atomic-force-microscope image of the surface of sample A, in which atomic steps are observed.

LSMO layer, as shown in previous reports on LAO/STO with a metallic capping layer [13,24,25]. As shown in Fig. 1(b), in which we assume that a simple parabolic band structure is split due to the Rashba effect, the spin current that is injected into the LAO/STO interface moves the outer and inner Fermi surfaces in opposite directions under the ferromagnetic resonance (FMR) condition, generating a charge current in the [1110] (x) direction. This effect induces the electromotive force (EMF) between the electrodes at the edges of the sample in the [1110] direction [Fig. 1(a)]. We note that the EMF includes a signal originating from LSMO that is induced by the microwave electric field, such as the galvanomagnetic effects (e.g., anomalous Hall effect and planar Hall effect), which should be separated from the IEE signal. For this purpose, we have grown two LSMO/LAO/STO samples with a $2 \mathrm{DEG}$ (sample A) and without a 2DEG (sample B); as shown in a previous study on MBE-grown LAO/STO films [26], a 2DEG is formed only when the composition ratio $c=(1-\delta) /(1+\delta)$ of $\mathrm{La}$ to $\mathrm{Al}$ in $\mathrm{La}_{(1-\delta)} \mathrm{Al}_{(1+\delta)} \mathrm{O}_{3}$ is below $0.97 \pm 0.03$. In samples A and B, $c$ was set at $83 \%$ and $101 \%$, respectively. Because the IEE is observed only in sample A, we can extract the pure IEE signal by comparing the results between samples $\mathrm{A}$ and $\mathrm{B}$. The thickness of 2 u.c. of the LAO layer is the most suitable to avoid the degradation of the film quality of the LSMO layer caused by the off-stoichiometry condition
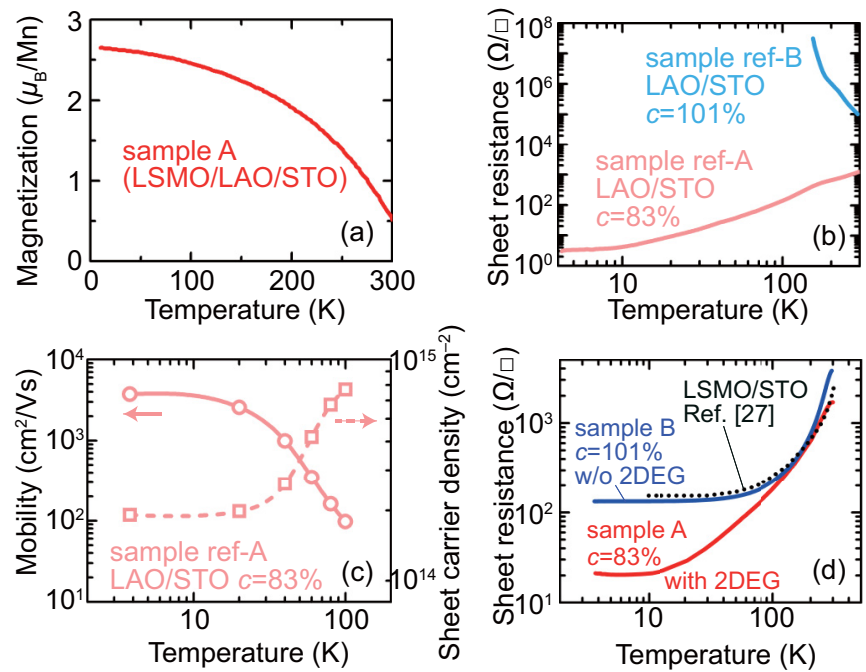

FIG. 2. (a) Temperature dependence of the magnetization of LSMO (30 u.c.)/LAO (2 u.c.)/STO (sample A). (b) Temperature dependence of the sheet resistance of the reference samples of LAO (8 u.c.)/STO with the ratio $c$ of $\mathrm{La}$ to $\mathrm{Al}$ of $83 \%$ (sample ref-A) and $101 \%$ (sample ref-B). (c) Temperature dependences of the mobility and the carrier density measured for sample ref-A $(c=83 \%)$. (d) Temperature dependence of the sheet resistance of the LSMO (30 u.c.)/LAO(2 u.c.)/STO samples with a $2 \mathrm{DEG}$ (sample A, $c=83 \%$ ) and without a $2 \mathrm{DEG}$ (sample $\mathrm{B}, c=101 \%$ ). The dotted curve is the sheet resistance reported for LSMO/STO, which is reproduced from Ref. [27] assuming the film thickness to be the same as that of our LSMO layer.

of LAO, especially in sample A. Generally, the (inverse) EE is known to tend to gradually decrease as the LAO thickness increases $[19,21]$. The scanning transmission electron microscope (STEM) image of sample A (LSMO/LAO/STO) shown in Fig. 1(c) confirms that all the layers are single crystalline and coherently grown on the STO substrate. The sample surface is atomically flat with atomic steps [Fig. 1(d)]. We measured the EMF in the [110] direction because this direction is nearly along the atomic step edges along which we can obtain the highest mobility [see Fig. 1(a)]. The LSMO layer has a Curie temperature above room temperature [Fig. 2(a)].

To confirm that a $2 \mathrm{DEG}$ is formed only when $c=83 \%$, we measured the transport properties of reference samples of $\mathrm{La}_{(1-\delta)} \mathrm{Al}_{(1+\delta)} \mathrm{O}_{3}$ (8 u.c. $\left.=3.2 \mathrm{~nm}\right) / \mathrm{STO}$ with $c=83 \%$ (named sample ref-A) and $c=101 \%$ (named sample ref-B), which were grown with the same growth conditions as those for samples A and B, respectively. Actually, as shown in Fig. 2(b), sample ref-A $(c=83 \%)$ shows metallic behavior while sample ref-B $(c=101 \%)$ shows insulating behavior, confirming that a 2DEG exists only when $c=83 \%$. By the Hall measurements, the sheet career density $n_{\mathrm{s}}$ of the 2DEG in sample ref-A was estimated to be $2.1 \times 10^{14} \mathrm{~cm}^{-2}$ and the mobility $\mu$ was $3.7 \times 10^{3} \mathrm{~cm}^{2} \mathrm{~V}^{-1} \mathrm{~s}^{-1}$ at $20 \mathrm{~K}$ [Fig. 2(c)]. As shown in Fig. 2(d), sample A shows similar metallic behavior, while sample B shows nearly the same temperature dependence of the sheet resistance as that of a single LSMO layer grown on STO (dotted curve reproduced from Ref. [27]). 


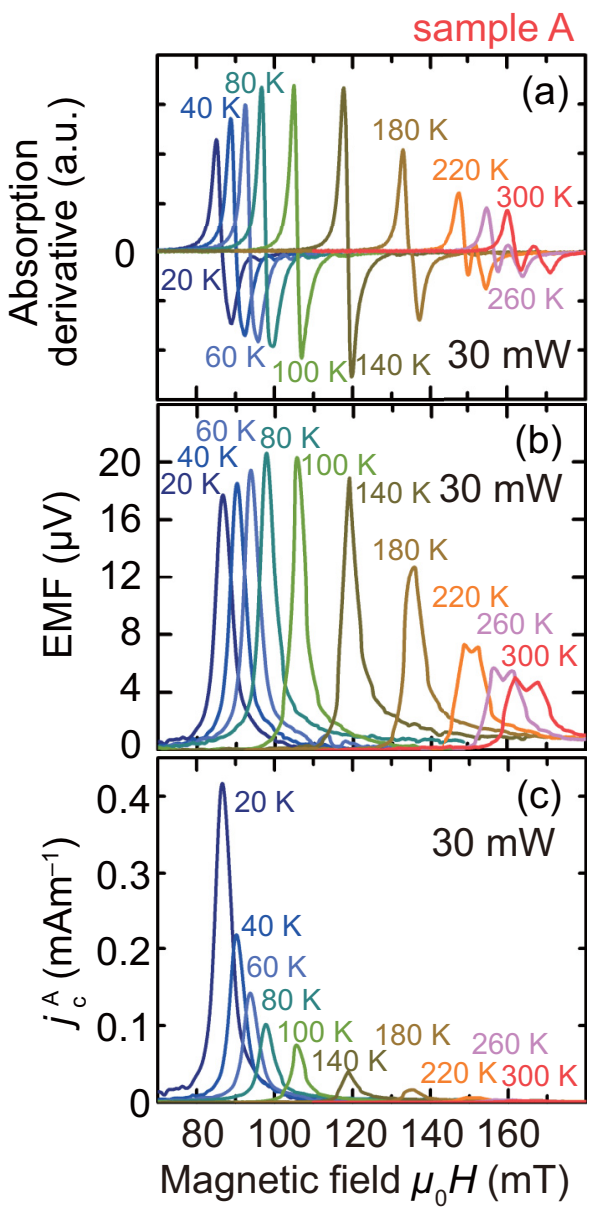

FIG. 3. (a)-(c) Magnetic-field $\mu_{0} H$ dependences of (a) the microwave absorption derivative, (b) EMF, and (c) $j_{\mathrm{c}}^{\mathrm{A}}$ measured for sample A (LSMO/LAO/STO with a 2DEG) at various temperatures. The used microwave power is $30 \mathrm{~mW}$.

These results confirm the presence of the 2DEG only in sample A.

We have carried out spin pumping measurements using a transverse electric $\left(\mathrm{TE}_{011}\right)$ cavity of an electron-spinresonance system with a microwave frequency of $9.1 \mathrm{GHz}$. We cut the samples into a small piece with a size of $2 \times 1 \mathrm{~mm}$, connected gold wires to the contacts at both edges of the sample with a distance of $1.5 \mathrm{~mm}$ apart, and put the sample at the center of the cavity. For the measurements, a static magnetic field $\mu_{0} H$ was applied along the [110] (y) direction in the film plane, which corresponds to the easy magnetization axis of LSMO. Meanwhile, the microwave magnetic field $h_{\mathrm{rf}}$ was applied along the [1110] direction. The used microwave power was $30 \mathrm{~mW}$.

As shown in Figs. 3(a) and 3(b), the EMF peak appears at the FMR magnetic field at all the measurement temperatures, indicating that the measured EMF is induced by the FMR, as in general spin pumping experiments. We note that we can eliminate the influence of the thermal effects as discussed in Sec. 1 of the Supplemental Material (SM) [28]. To derive the IEE signal from the EMF, we extracted the symmetric component $V_{\mathrm{S}}$, which includes the IEE signal, from the EMF$H$ curves (Sec. 2 in SM [28]). Then, we estimated the sheet (a)

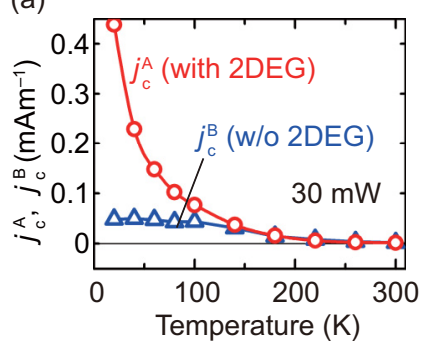

(b) sample A with 2DEG

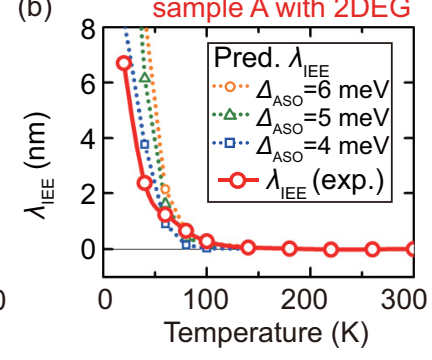

(c)

(d) $\quad\left|v_{x}\right|(\mathrm{au}) 0=0.7$

(e) $\quad \Delta_{\mathrm{ASO}}=5 \mathrm{meV}$
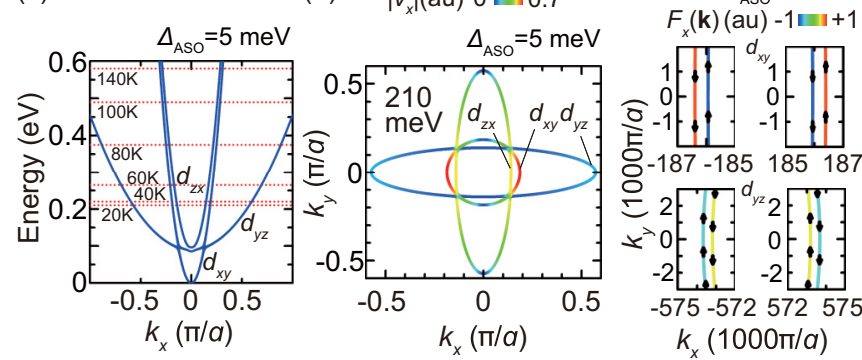

FIG. 4. (a) Comparison between $j_{\mathrm{c}}^{\mathrm{A}}$ (with a 2DEG) and $j_{\mathrm{c}}^{\mathrm{B}}$ (without a 2DEG) as a function of temperature. (b) Temperature dependences of experimental $\lambda_{\mathrm{IEE}}$ and predicted $\lambda_{\mathrm{IEE}}$ for $\Delta_{\mathrm{ASO}}=4$, 5 , and $6 \mathrm{meV}$. (c) Dispersion relation of the 2DEG at the LAO/STO interface (blue curves). The red dotted lines, from bottom to top, are the estimated $E_{\mathrm{F}}$ positions at $20,40,60,80,100$, and $140 \mathrm{~K}$ in our sample. (d) Fermi surface of the 2DEG when the carrier density is $2.1 \times 10^{14} \mathrm{~cm}^{-2}$ and $E_{\mathrm{F}}=210 \mathrm{meV}$, which corresponds to the measurement condition of the IEE at $20 \mathrm{~K}$. The color scale represents the absolute value of the group velocity in the $x$ direction. (e) Details of the Fermi surface with the spin orientations (see arrows). The color scale represents $F_{x}(\mathbf{k})$, which is an indicator of the contribution of each state to the electron flow when a spin current is injected. In (c)-(e), $\Delta_{\text {AsO }}$ is assumed to be $5 \mathrm{meV}$. Note the large difference in the scale of the axes between (d) and (e).

current density $j_{\mathrm{c}}^{\mathrm{A}}=V_{\mathrm{s}} /(w R)$, where $R$ is the resistance [see Fig. 2(d)] and $w$ is the sample width $(1 \mathrm{~mm})$. In Fig. 3(c), one can see a drastic increase in $j_{\mathrm{c}}^{\mathrm{A}}$ with decreasing temperature.

To separate the IEE signal from the one originating from LSMO such as the galvanomagnetic effects, we derived the IEE-induced sheet current density $j_{\mathrm{c}}^{2 \mathrm{D}}$ by subtracting the sheet current density $j_{\mathrm{c}}^{\mathrm{B}}$, which was measured for sample B (see Sec. 3 of SM [28]), from $j_{\mathrm{c}}^{\mathrm{A}}$. As shown in Fig. 4(a), $j_{\mathrm{c}}^{\mathrm{A}}$ is much larger than $j_{\mathrm{c}}^{\mathrm{B}}$, especially at low temperatures, indicating that $j_{\mathrm{c}}^{\mathrm{A}}$ is mainly attributed to the IEE signal.

We estimated the spin current density $j_{\mathrm{s}}$ in sample A using

$j_{\mathrm{s}}=\frac{g^{\uparrow \downarrow} \gamma^{2}\left(\mu_{0} h_{\mathrm{rf}}\right)^{2} \hbar\left(4 \pi M_{s} \gamma+\sqrt{\left.\left(4 \pi M_{s} \gamma\right)^{2}+4 \omega^{2}\right)}\right.}{8 \pi \alpha_{\mathrm{A}}^{2}\left[\left(4 \pi M_{s} \gamma\right)^{2}+4 \omega^{2}\right]}\left(\frac{2 e}{\hbar}\right)$,

where $\hbar$ is the Dirac constant, $\omega$ is the angular frequency of the microwave, $e$ is the elementary charge, $h_{\mathrm{rf}}$ is the microwave magnetic field, $\gamma$ is the gyromagnetic ratio in LSMO, $\alpha_{\mathrm{A}}$ is the Gilbert damping constant, and $M_{s}$ is the saturation magnetization of LSMO [29]. $g^{\uparrow \downarrow}$ is the real part of the spin-mixing conductance given by

$$
g^{\uparrow \downarrow}=\frac{4 \pi M_{s} d_{\mathrm{LSMO}}\left(\alpha_{\mathrm{A}}-\alpha_{\mathrm{i}}\right)}{\hbar \gamma \mu_{0}},
$$



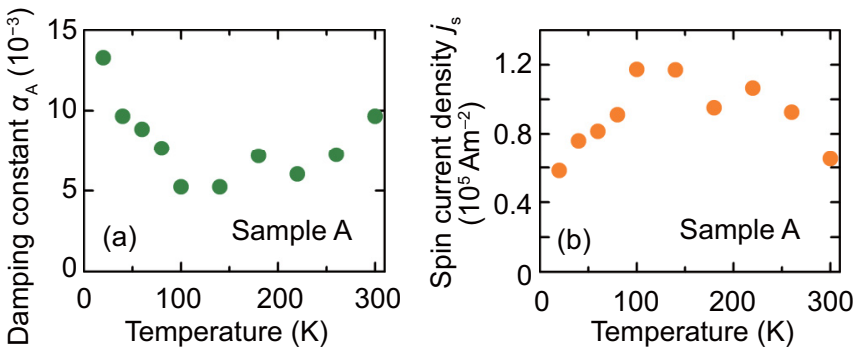

FIG. 5. (a) Obtained damping constant $\alpha_{\mathrm{A}}$ and (b) $j_{\mathrm{s}}$ as a function of temperature.

where $d_{\text {LSMO }}$ is the thickness of LSMO $(12 \mathrm{~nm})$, and $\alpha_{\mathrm{i}}$ is the intrinsic Gilbert damping constant of LSMO with no spin current generation. Due to the spin current generation from LSMO in sample $\mathrm{A}, \alpha_{\mathrm{A}}$ is larger than $\alpha_{\mathrm{i}} \cdot \alpha_{\mathrm{A}}$ and $\alpha_{\mathrm{i}}$ are obtained by

$$
\alpha_{\xi}=\frac{\sqrt{3}}{2} \frac{g \mu_{\mathrm{B}}}{2 \pi f} \frac{1}{\hbar} \Delta W_{\xi} \quad(\xi=A, i),
$$

where $f$ is the frequency of the microwave, $\mu_{\mathrm{B}}$ is the Bohr magneton, and $g$ is the effective electron $g$-factor (1.95 for LSMO [30]). $\Delta W_{\mathrm{A}}$ and $\Delta W_{\mathrm{i}}$ are the experimental FMR spectral linewidths for sample A and intrinsic LSMO (e.g., no spin injection), respectively. The obtained temperature dependence of $\alpha_{\mathrm{A}}$ is shown in Fig. 5(a). Here, we set $\alpha_{\mathrm{i}}$ to be $1.57 \times 10^{-3}$, which was reported for a high-quality LSMO film in Ref. [31]. Depending on the difference of the crystal quality of the LSMO layers, this method may overestimate $j_{\mathrm{s}}$ and thus underestimate $\lambda_{\text {IEE }}$ in our study. We have obtained $g^{\uparrow \downarrow}=40 \mathrm{~nm}^{-2}$ and $j_{\mathrm{s}}=5.8 \times 10^{4} \mathrm{~A} \mathrm{~m}^{-2}$ at $20 \mathrm{~K}$. The obtained temperature dependence of $j_{\mathrm{s}}$ is shown in Fig. 5(b). The complicated temperature dependences are likely related to the changes in the spin polarization and the magnetization of LSMO as a function of temperature.

Following the above-mentioned procedure, we obtained the temperature dependence of $\lambda_{\text {IEE }}\left(=j_{\mathrm{c}}^{2 \mathrm{D}} / j_{s}\right)$ [red open circles in Fig. 4(b)], which shows that $\lambda_{\text {IEE }}$ drastically increases with decreasing temperature and amounts up to $+6.7 \mathrm{~nm}$ at $20 \mathrm{~K}$. This temperature dependence is similar to that in Ref. [21] but is completely opposite to that reported in Refs. [19,20]. As discussed below, this characteristic increase in $\lambda_{\text {IEE }}$ with decreasing temperature mainly originates from the intrinsic feature of the IEE in the LAO/STO system.

Following the approach in Ref. [32], we calculated the band structure of the LAO/STO interface using the effectivemass Hamiltonian with atomic spin-orbit coupling and interorbital nearest-neighbor hopping by polar lattice distortion based on the six $3 d-t_{2 g}$ orbitals of up- and down-spin components of the $d_{x y}, d_{y z}$, and $d_{z x}$ orbitals of Ti (see Sec. 4 in SM [28]). The calculated band structure is shown in Fig. 4(c). Comparing the $n_{\mathrm{s}}$ values obtained for sample ref-A [see Fig. 2(c)] and the theoretical carrier density (Fig. S4 in SM [28]), we estimated the $E_{\mathrm{F}}$ positions in our samples, which are shown as the red dotted lines in Fig. 4(c). From the Boltzmann equation, for the $n$th Fermi surface $\mathrm{SF}_{n}$, the two-dimensional (2D) current density $j_{\mathrm{c}} \mathrm{FS}_{n}$ and the nonequilibrium spin density $\delta s^{\mathrm{FS}_{n}}$ are expressed by

$j_{\mathrm{c}}^{\mathrm{FS}_{n}}=\frac{e^{2}}{4 \pi^{2} \hbar} \int^{\mathrm{FS}_{n}} F_{x}(\mathbf{k}) d S_{\mathrm{F}}, \delta s^{\mathrm{FS}_{n}}=\frac{e}{4 \pi^{2} \hbar} \int^{\mathrm{FS}_{n}}\left|S_{y}(\mathbf{k})\right| d S_{\mathrm{F}}$,

where $e$ is the free electron charge, $\hbar$ is the Dirac constant, and $d S_{\mathrm{F}}$ is the infinitesimal area (=length in two dimensions) of the Fermi surface [33]. Here, the $x$ and $y$ axes are set along the [100] and [010] directions in this theoretical discussion, respectively. To avoid the complexity of the calculation due to the complex spin structure around the $\langle 110\rangle$ directions [17], we assumed the directions of the charge current along [100] and spin of the injected spin current along [010] for simplicity. Here, $F_{x}(\mathbf{k})$ and $S_{y}(\mathbf{k})$ are defined as

$$
\begin{aligned}
& F_{x}(\mathbf{k})=F \operatorname{sgn}\left[S_{y}(\mathbf{k})\right] \tau(\mathbf{k}) v_{x}(\mathbf{k}) \frac{v_{x}(\mathbf{k})}{|\mathbf{v}(\mathbf{k})|}, \\
& S_{y}(\mathbf{k})=F \tau(\mathbf{k}) \sigma_{y}(\mathbf{k}) \frac{v_{x}(\mathbf{k})}{|\mathbf{v}(\mathbf{k})|},
\end{aligned}
$$

where $F$ is the absolute value of the effective electric field that is applied to each electron state, and $v_{x}(\mathbf{k})$ is the $x$ direction component of the group velocity $\mathbf{v}(\mathbf{k})$. We assumed that the relaxation time $\tau(\mathbf{k})$ is proportional to $|\mathbf{k}|$. Then, $j_{\mathrm{c}}^{2 \mathrm{D}}$, the total nonequilibrium spin density $\delta s$, and $\lambda_{\text {IEE }}$ are expressed by

$$
j_{\mathrm{c}}^{2 \mathrm{D}}=\sum_{n} j_{\mathrm{c}}^{\mathrm{FS} n}, \quad \delta s=\sum_{n} \delta s^{\mathrm{FS} n}, \quad \lambda_{\mathrm{IEE}}=\frac{j_{\mathrm{c}}^{2 \mathrm{D}}}{j_{\mathrm{s}}}=\frac{\tau}{e} \frac{j_{\mathrm{c}}^{2 \mathrm{D}}}{\delta s} .
$$

The most important indication in the above equations is that the charge current is mainly carried by electrons with large $v_{x}(\mathbf{k})$. As shown in the calculated $v_{x}(\mathbf{k})$ mapping at the Fermi surface when $E_{\mathrm{F}}=210 \mathrm{meV}$ [Fig. 4(d)], which corresponds to the case of the measurement temperature of $20 \mathrm{~K}$ in our study, we see that electrons in the vicinity of $k_{y}=0$ mainly contribute to the charge current. In fact, especially the $d_{x y}$ states that are located at $k_{x} \cong \pm 0.186 \pi / a$ have large $v_{x}(\mathbf{k})$ and $F_{x}(\mathbf{k})$ as shown in Figs. 4(d) and 4(e), where $a$ is a lattice constant of STO. Here, the atomic spinorbit energy $\Delta_{\text {AsO }}$ was assumed to be $5 \mathrm{meV}$, which corresponds to the effective Rashba parameter $\alpha_{\mathrm{R}}=\hbar^{2} \Delta k /(2 m)$ of $3.5 \times 10^{-13} \mathrm{eV} \mathrm{m}$ for the $d_{x y}$ band with the effective mass $m=0.41 m_{0}$ ( $m_{0}$ is the electron mass in a vacuum) [32]. Here, $\Delta k$ is the spin split of $k_{x}$ when $k_{y}=0$ [see Fig. 4(e)]. This relatively small value of $\alpha_{\mathrm{R}}$ is due to the large $E_{\mathrm{F}}$ (or carrier density) in our sample. If we can decrease $E_{\mathrm{F}}$ down to the Lifshitz point $\left(E_{\mathrm{F}} \approx 100 \mathrm{meV}\right), \Delta k$ and thus $\alpha_{\mathrm{R}}$ will be largely increased, as described in Sec. 6 in SM [28]. Similarly, when $E_{F} \geqslant 210 \mathrm{meV}$, the $d_{x y}$ states near $k_{y}=0$ have a dominant contribution to the charge current, leading to a nearly energy-independent value of $j_{\mathrm{c}}^{2 \mathrm{D}} / \delta s\left(=e \lambda_{\mathrm{IEE}} / \tau\right)$ when $E_{\mathrm{F}} \geqslant 210 \mathrm{meV}$ and $\Delta_{\mathrm{ASO}} \cong 5 \mathrm{meV}$ (Fig. 6). Thus, $\lambda_{\text {IEE }}$ is almost determined by $\tau$ in this energy region.

In a way similar to the derivation of Eqs. (4)-(6), we can obtain the relaxation time $\tau$ from the experimental sheet resistance of sample A shown in Fig. 2(d) (Sec. 5 in SM [28]). Using the theoretical value of $j_{\mathrm{c}}^{2 \mathrm{D}} / \delta s$ (Fig. 6) and $\tau$, we can predict $\lambda_{\text {IEE }}$ that is expected in our system at each temperature [dotted curves in Fig. 4(b)]. We see that predicted $\lambda_{\text {IEE }}$ increases with decreasing temperature as with the experimental $\lambda_{\text {IEE}}$, which confirms that our result originates 


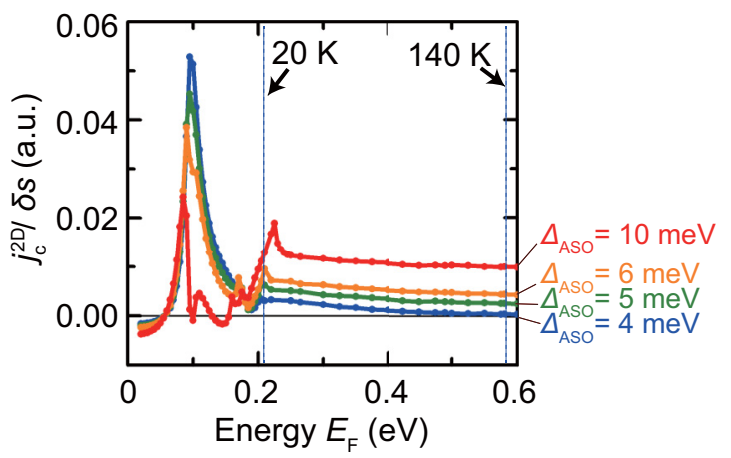

FIG. 6. Calculated $j_{\mathrm{c}}^{2 \mathrm{D}} / \delta s$ as a function of $E_{\mathrm{F}}$ when $\Delta_{\mathrm{ASO}}$ is 4 , 5,6 , and $10 \mathrm{meV}$. The dotted vertical lines correspond to the carrier concentrations at 20 and $140 \mathrm{~K}$ in our sample (see Fig. S4 in SM [28]). We can see that $j_{\mathrm{c}}^{2 \mathrm{D}} / \delta s$ can be negative for $\Delta_{\mathrm{ASO}}=10 \mathrm{meV}$, which may be the origin of the negative $\lambda_{\text {IEE }}$ reported in Ref. [13].

from the intrinsic IEE. The reason for the larger values of predicted $\lambda_{\text {IEE }}$ than the experimental values especially at low temperatures is probably due to our overestimation of $j_{s}$ or the small influence of spin scattering.

Comparing between the previous results of the IEE at LAO/STO [13,19-21] and our result, the small thickness of LAO only of 2 u.c. and the single crystallinity of our sample are likely keys to suppressing the extrinsic effect and to obtaining the large intrinsic IEE. Furthermore, our band-structure calculation suggests that $\lambda_{\text {IEE }}$ will be dramatically enhanced if we can tune the $E_{\mathrm{F}}$ position at around the Lifshitz point (Fig. 6). At the same time, we see that increasing $\tau$ is important to enhance the IEE, indicating that single-crystalline $2 \mathrm{D}$ systems with a high mobility are very promising for efficient conversion between spin and charge currents.

In summary, we have demonstrated efficient intrinsic spinto-charge current conversion in a two-dimensional electron gas using an all-epitaxial single-crystal heterostructure of $\mathrm{LSMO} / \mathrm{LAO} / \mathrm{STO}$. With decreasing temperature to $20 \mathrm{~K}$, $\lambda$ IEE was drastically enhanced to $+6.7 \mathrm{~nm}$. Our band-structure calculation well reproduces this behavior and predicts a further significant enhancement of $\lambda_{\text {IEE }}$ by adjusting the Fermi-level position near the Lifshitz point [18,34-39].

This work is partly supported by Grants-in-Aid for Scientific Research by MEXT (Grant No. 18H03860), CREST of JST (Grant No. JPMJCR1777), Advanced Characterization Nanotechnology Platform of the University of Tokyo by MEXT, and the Spintronics Research Network of Japan (Spin-RNJ). We thank K. Takeshima for technical help for the MBE growth and electron spin resonance measurements.
[1] F. Oboril, R. Bishnoi, M. Ebrahimi, and M. B. Tahoori, IEEE Trans. Comput.-Aided Design Integr. Circuits Syst. 34, 367 (2015).

[2] E. Saitoh, M. Ueda, H. Miyajima, and G. Tatara, Appl. Phys. Lett. 88, 182509 (2006).

[3] A. Azevedo, L. H. Vilela-Leão, R. L. Rodríguez-Suárez, A. F. Lacerda Santos, and S. M. Rezende, Phys. Rev. B 83, 144402 (2011).

[4] K. Ando, S. Takahashi, J. Ieda, Y. Kajiwara, H. Nakayama, T. Yoshino, K. Harii, Y. Fujikawa, M. Matsuo, S. Maekawa, and E. Saitoh, J. Appl. Phys. 109, 103913 (2011).

[5] J. C. R. Sánchez, L. Vila, G. Desfonds, S. Gambarelli, J. P. Attané, J. M. De Teresa, C. Magén, and A. Fert, Nat. Commun. 4, 2944 (2013).

[6] P. Noel, C. Thomas, Y. Fu, L. Vila, B. Haas, P-H. Jouneau, S. Gambarelli, T. Meunier, P. Ballet, and J. P. Attané, Phys. Rev. Lett. 120, 167201 (2018).

[7] S. Oyarzún et al., Nat. Commun. 7, 13857 (2016).

[8] J.-C. Rojas-Sánchez et al., Phys. Rev. Lett. 116, 096602 (2016).

[9] J.-C. Rojas-Sánchez and A. Fert, Phys. Rev. Applied 11, 054049 (2019).

[10] Ka Shen, G. Vignale, and R. Raimondi, Phys. Rev. Lett. 112, 096601 (2014).

[11] V. M. Edelstein, Solid State Commun. 73, 233 (1990).

[12] D. C. Vaz et al., Nat. Mater. 18, 1187 (2019).

[13] E. Lesne, Y. Fu, S. Oyarzun, J. C. Rojas-Sánchez, D. C. Vaz, H. Naganuma, G. Sicoli, J. P. Attané, M. Jamet, E. Jacquet, J. M. George, A. Barthélémy, H. Jaffrès, A. Fert, M. Bibes, and L. Vila, Nat. Mater. 15, 1261 (2016).
[14] A. Ohtomo and H. Y. Hwang, Nature (London) 427, 423 (2004).

[15] S. Gariglio, M. Gabay, and J.-M. Triscone, APL Mater. 4, 060701 (2016).

[16] A. D. Caviglia, M. Gabay, S. Gariglio, N. Reyren, C. Cancellieri, and J. M. Triscone, Phys. Rev. Lett. 104, 126803 (2010).

[17] P. D. C. King, S. M. K. Walker, A. Tamai, A. De La Torre, T. Eknapakul, P. Buaphet, S. K. Mo, W. Meevasana, M. S. Bahramy, and F. Baumberger, Nat. Commun. 5, 3414 (2014).

[18] H. Liang, L. Cheng, L. Wei, Z. Luo, G. Yu, C. Zeng, and Z. Zhang, Phys. Rev. B 92, 075309 (2015).

[19] Q. Song, H. Zhang, T. Su, W. Yuan, Y. Chen, W. Xing, J. Shi, J. Sun, and W. Han, Sci. Adv. 3, e1602312 (2017).

[20] Y. Wang, R. Ramaswamy, M. Motapothula, K. Narayanapillai, D. Zhu, J. Yu, T. Venkatesan, and H. Yang, Nano Lett. 17, 7659 (2017).

[21] J. Y. Chauleau, M. Boselli, S. Gariglio, R. Weil, G. De Loubens, J. M. Triscone, and M. Viret, Europhys. Lett. 116, 17006 (2016).

[22] W. Zhang, W. Han, X. Jiang, S. H. Yang, and S. S. P. Parkin, Nat. Phys. 11, 496 (2015).

[23] J.-C. Rojas-Sánchez, N. Reyren, P. Laczkowski, W. Savero, J. P. Attané, C. Deranlot, M. Jamet, J.-M. George, L. Vila, and H. Jaffrès, Phys. Rev. Lett. 112, 106602 (2014).

[24] N. Nakagawa, H. Y. Hwang, and D. A. Muller, Nat. Mater. 5, 204 (2006).

[25] E. Lesne, N. Reyren, D. Doennig, R. Mattana, H. Jaffrès, V. Cros, F. Petroff, F. Choueikani, P. Ohresser, R. Pentcheva, A. Barthélémy, and M. Bibes, Nat. Commun. 5, 4291 (2014). 
[26] M. P. Warusawithana, C. Richter, J. A. Mundy, P. Roy, J. Ludwig, S. Paetel, T. Heeg, A. A. Pawlicki, L. F. Kourkoutis, M. Zheng, M. Lee, B. Mulcahy, W. Zander, Y. Zhu, J. Schubert, J. N. Eckstein, D. A. Muller, C. S. Hellberg, J. Mannhart, and D. G. Schlom, Nat. Commun. 4, 2351 (2013).

[27] H. Boschker, J. Verbeeck, R. Egoavil, S. Bals, G. van Tendeloo, M. Huijben, E. P. Houwman, G. Koster, D. H. A. Blank, and G. Rijnders, Adv. Funct. Mater. 22, 2235 (2012).

[28] See Supplemental Material at http://link.aps.org/supplemental/ 10.1103/PhysRevResearch.2.012014 for the influence of the thermal effects, derivation of the symmetric component of the EMF- $H$ curves, spin pumping data of sample B, and details of the calculations.

[29] K. Ando and E. Saito, J. Appl. Phys. 108, 113925 (2010).

[30] H. K. Lee, I. Barsukov, A. G. Swartz, B. Kim, L. Yang, H. Y. Hwang, and I. N. Krivorotov, AIP Adv. 6, 055212 (2016).
[31] G. Y. Luo, M. Belmeguenai, Y. Roussigné, C. R. Chang, J. G. Lin, and S. M. Chérif, AIP Adv. 5, 097148 (2015).

[32] Y. Kim, R. M. Lutchyn, and C. Nayak, Phys. Rev. B 87, 245121 (2013).

[33] P. Gambardella and I. M. Miron, Philos. Trans. R. Soc., A 369, 3175 (2011).

[34] I. Pallecchi, M. Codda, E. Galleani d'Agliano, D. Marré, A. D. Caviglia, N. Reyren, S. Gariglio, and J.-M. Triscone, Phys. Rev. B 81, 085414 (2010).

[35] H. Kozuka, K. Ohbayashi, and K. Koumoto, Sci. Technol. Adv. Mater. 16, 026001 (2015).

[36] C. T. Bui and F. Rivadulla, Phys. Rev. B 90, 100403(R) (2014).

[37] B. W. Wu, G. Y. Luo, J. G. Lin, and S. Y. Huang, Phys. Rev. B 96, 060402(R) (2017).

[38] J. Lustikova, Y. Shiomi, and E. Saitoh, Phys. Rev. B 92, 224436 (2015).

[39] Y. X. Liu, D. Z.-Y. Ting, and T. C. McGill, Phys. Rev. B 54, 5675 (1996). 